Supporting Information Kirsch, Overman

\title{
Catalytic Asymmetric Intramolecular Aminopalladation: Improved Palladium(II) Catalysts
}

\author{
Stefan F. Kirsch, Larry E. Overman* \\ Department of Chemistry, 516 Rowland Hall, University of California, Irvine, CA 92697-2025
}

\section{Supporting Information}

Copies of ${ }^{1} \mathrm{H}$ and ${ }^{13} \mathrm{C}$ NMR spectra for 2-oxazolidinones 6, 8, 10 and 12; copies of HPLC traces to determine enantiopurity of $\mathbf{6 , 8}, 10$ and 12 .

(10 Pages)

General experimental details: All HPLC analyses used to determine enantiomeric purity were calibrated with samples of the racemate. All reactions were performed under Argon. $\mathrm{CH}_{2} \mathrm{Cl}_{2}$ was dried according to published procedures. ${ }^{1}$ COP-OAc, di- $\mu$-acetatobis[ $\eta^{5}-(S)-(\mathrm{p} R)-2-\left(2^{\prime}-\left(4^{\prime}-\right.\right.$ methylethyl)oxazolinyl)cyclopentadienyl,1-C,3'- $N)\left(\eta^{4}\right.$-tetraphenylcyclobutadiene)cobalt] dipalladium [(+)-4] was prepared according to published procedures. ${ }^{2}$

\footnotetext{
${ }^{1}$ a) Pangborn, A. B.; Giardello, M. A.; Grubbs, R. H.; Rosen, R. K.; Timmers, F. J. Organometallics 1996, 15, 1518. b) GlassContour Homepage: http://www.glasscontour.com.

${ }^{2}$ (a) Anderson, C. E.; Kirsch, S. F.; Overman, L. E.; Richards, C. J.; Watson, M. P. Org. Synth. submitted for publication; available from the senior author upon request. (b) Stevens, A. M.; Richards, C. J. Organometallics 1999, $18,1346$.
} 


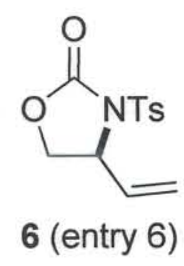

sk238

$1 \mathrm{H}$ spectrum

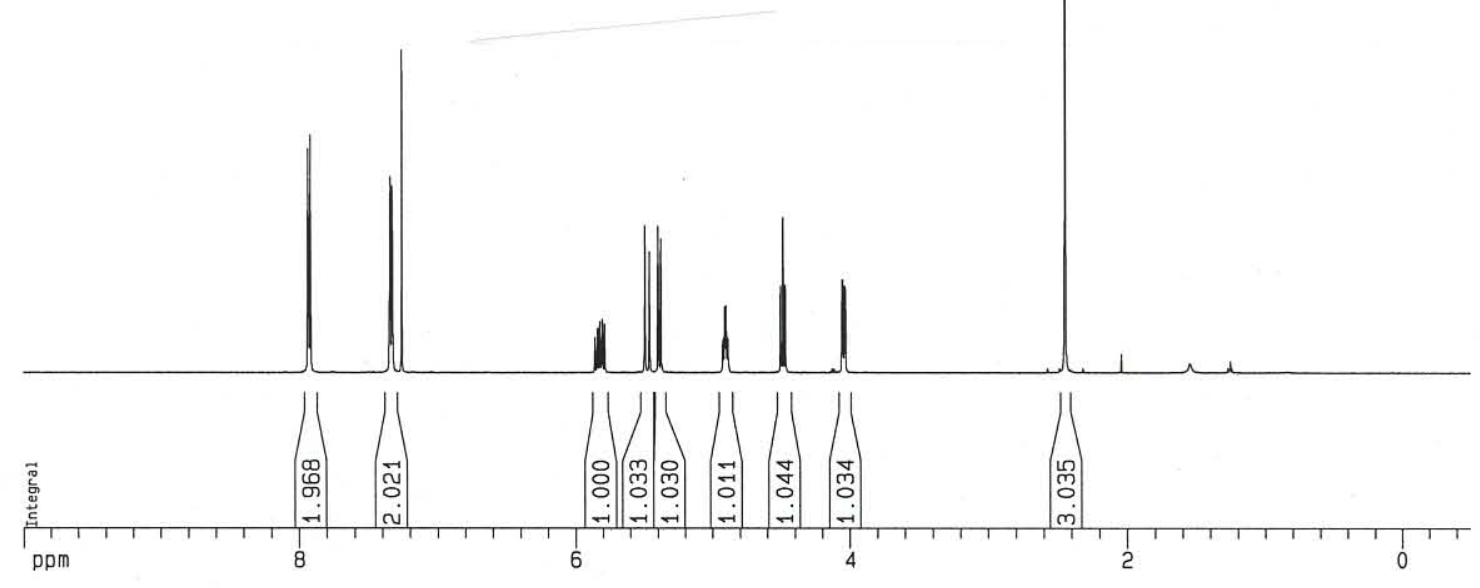

a
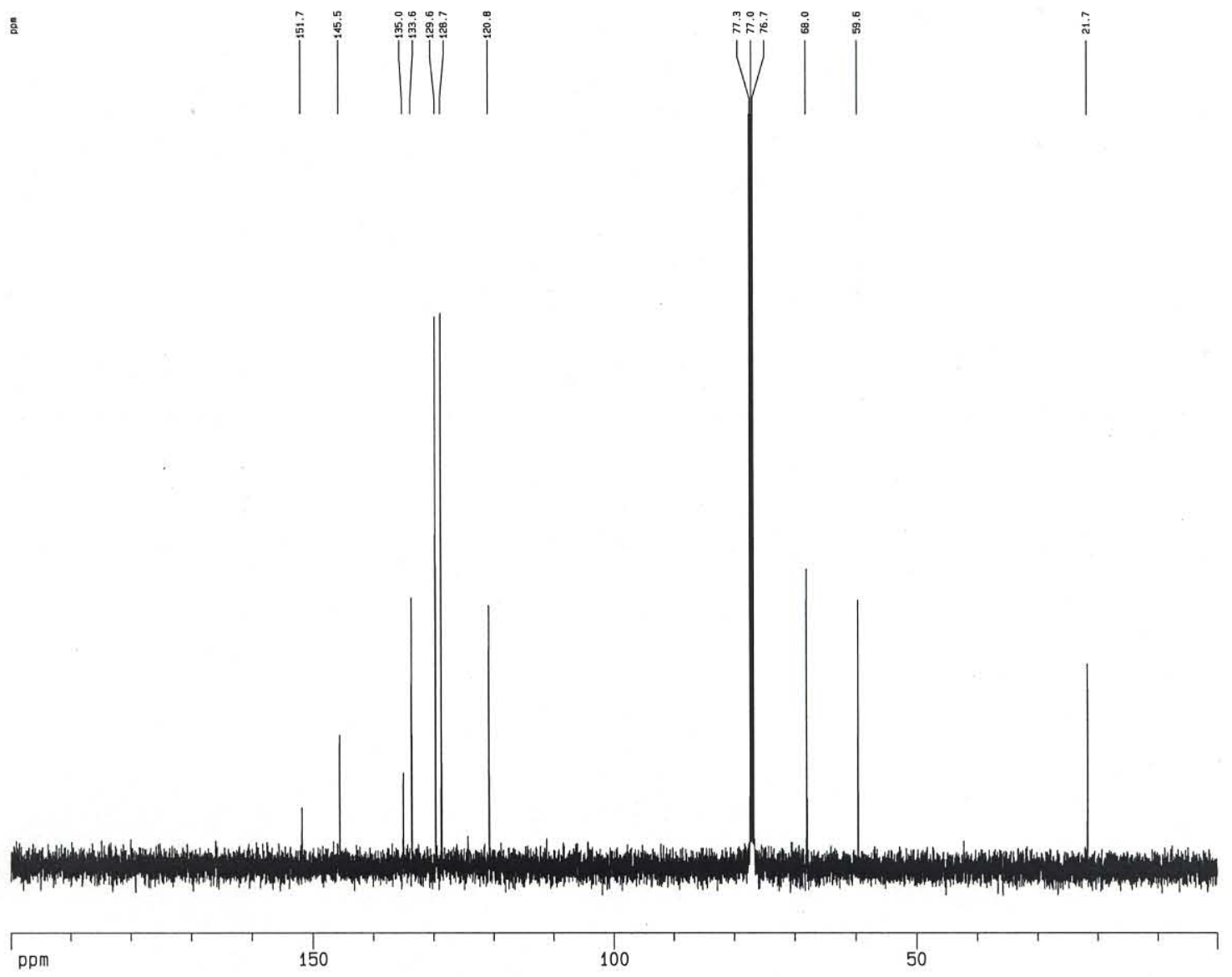


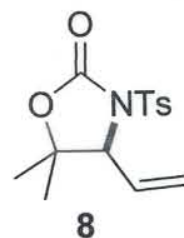

sk259

$1 \mathrm{H}$ spectrum
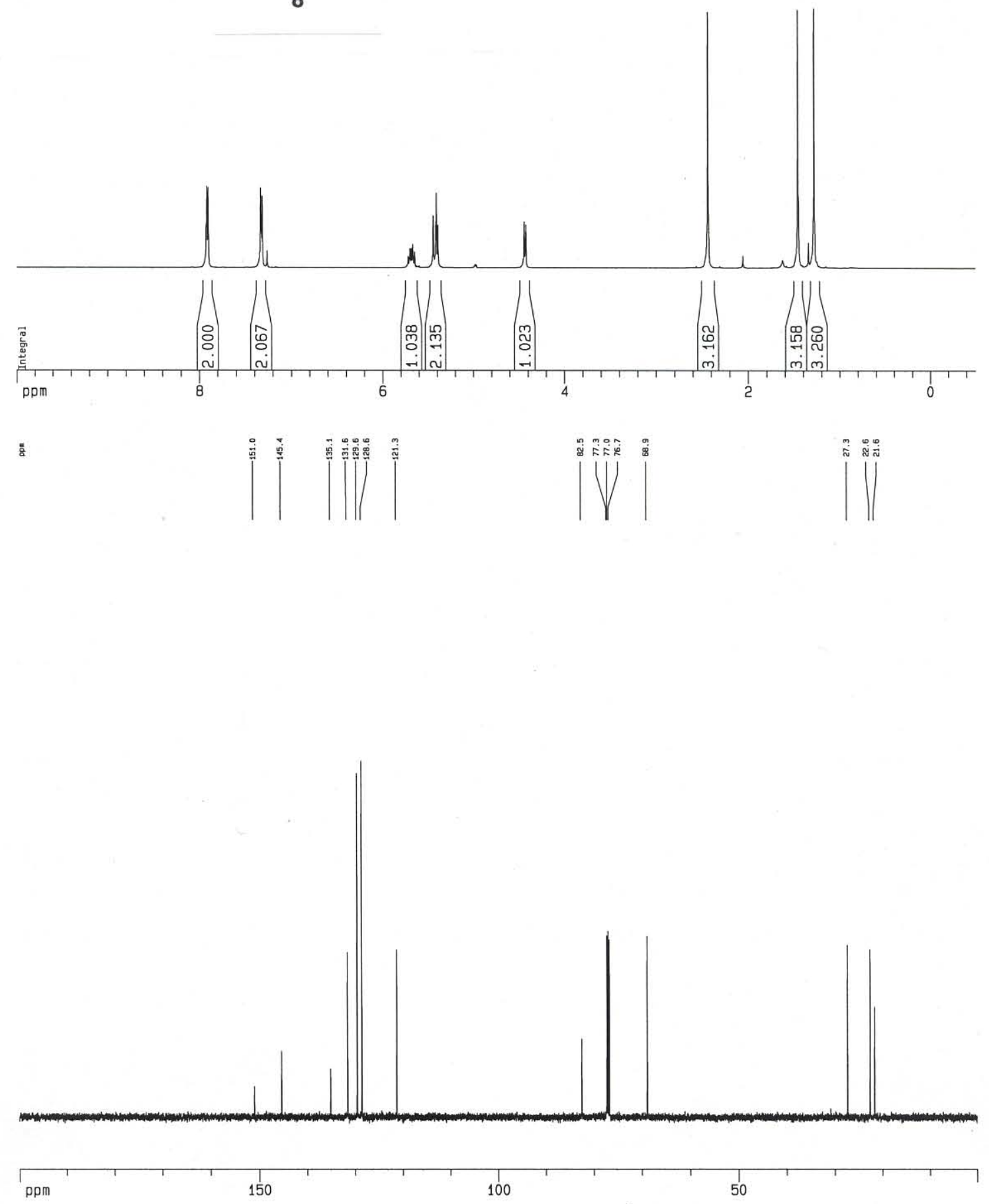


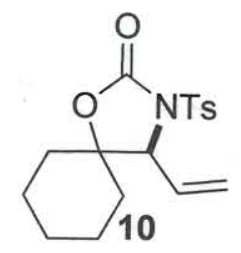

sk257

$1 \mathrm{H}$ spectrum
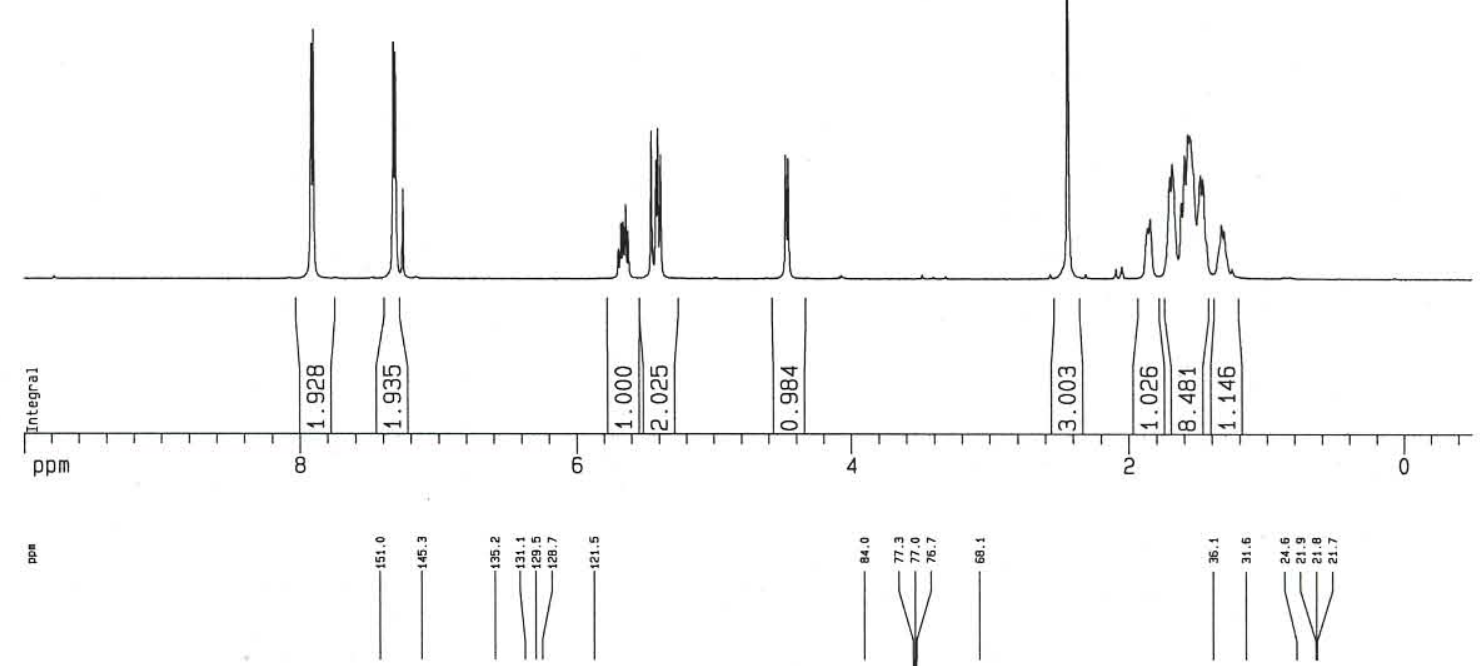

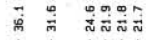

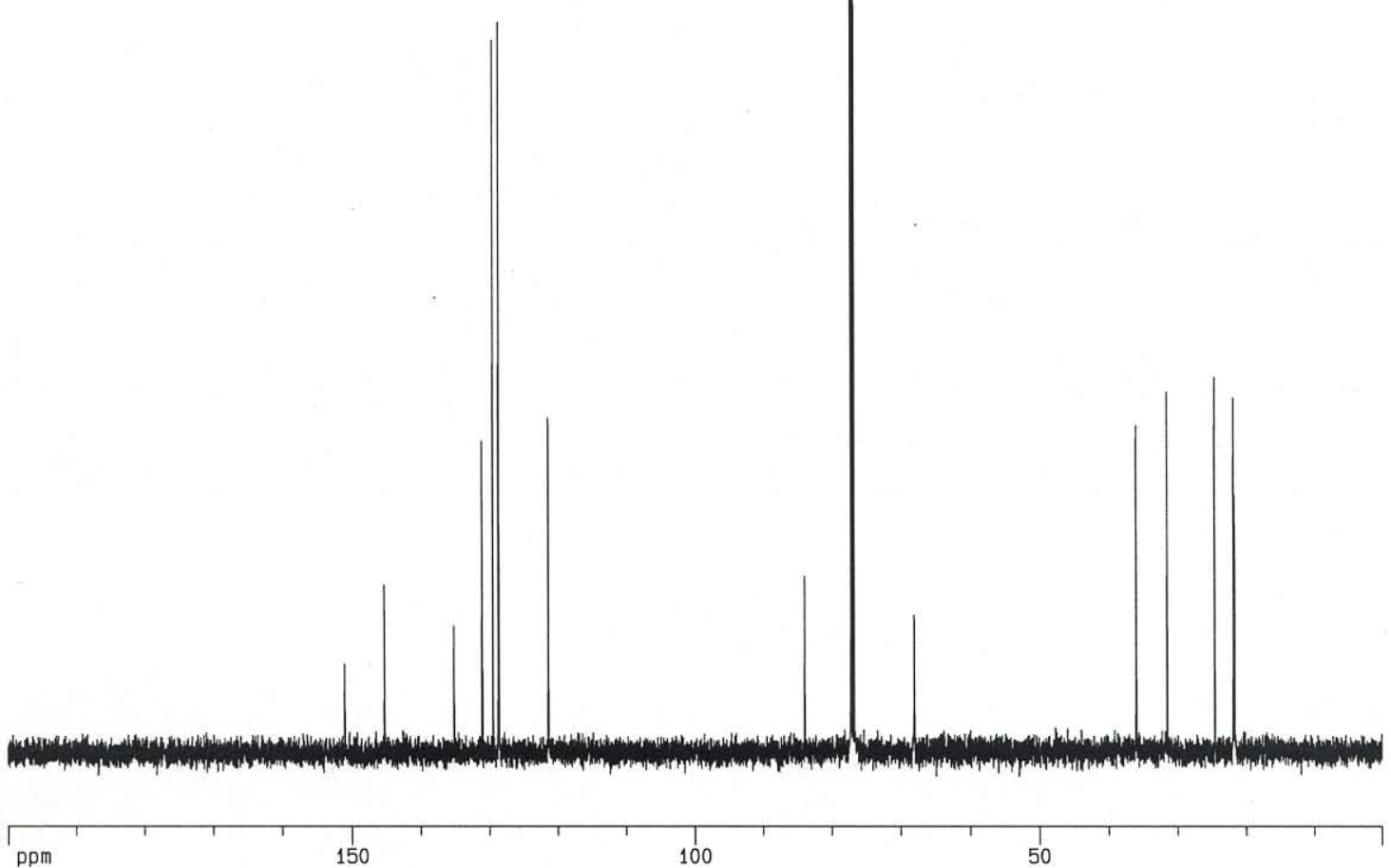




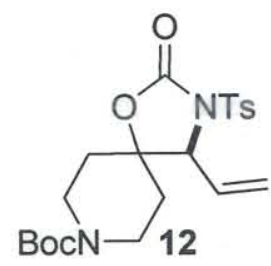

sk264

$1 \mathrm{H}$ spectrum

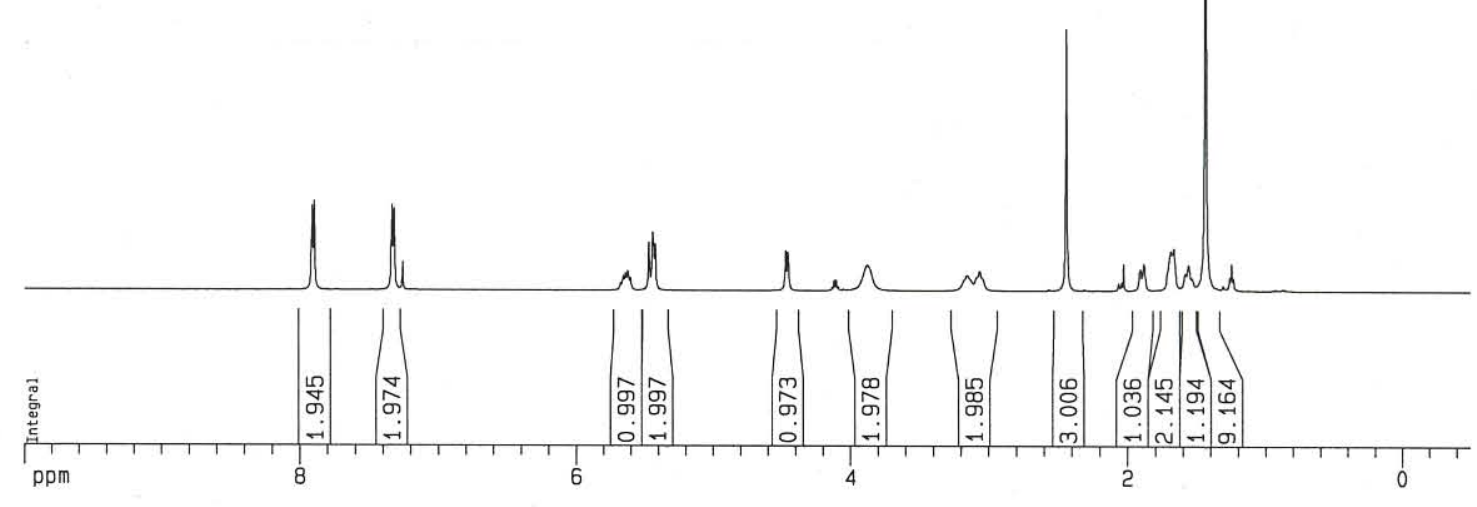

:
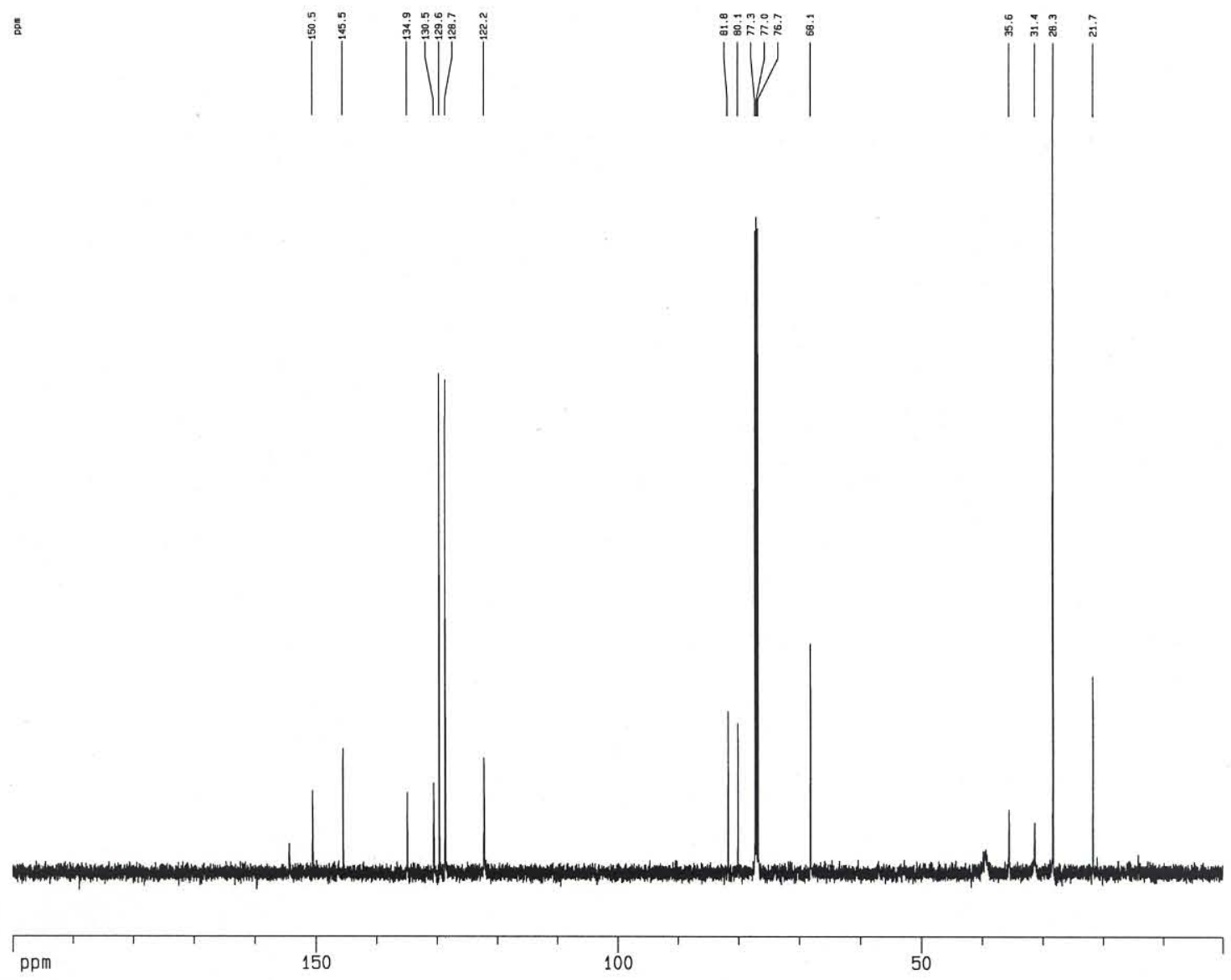

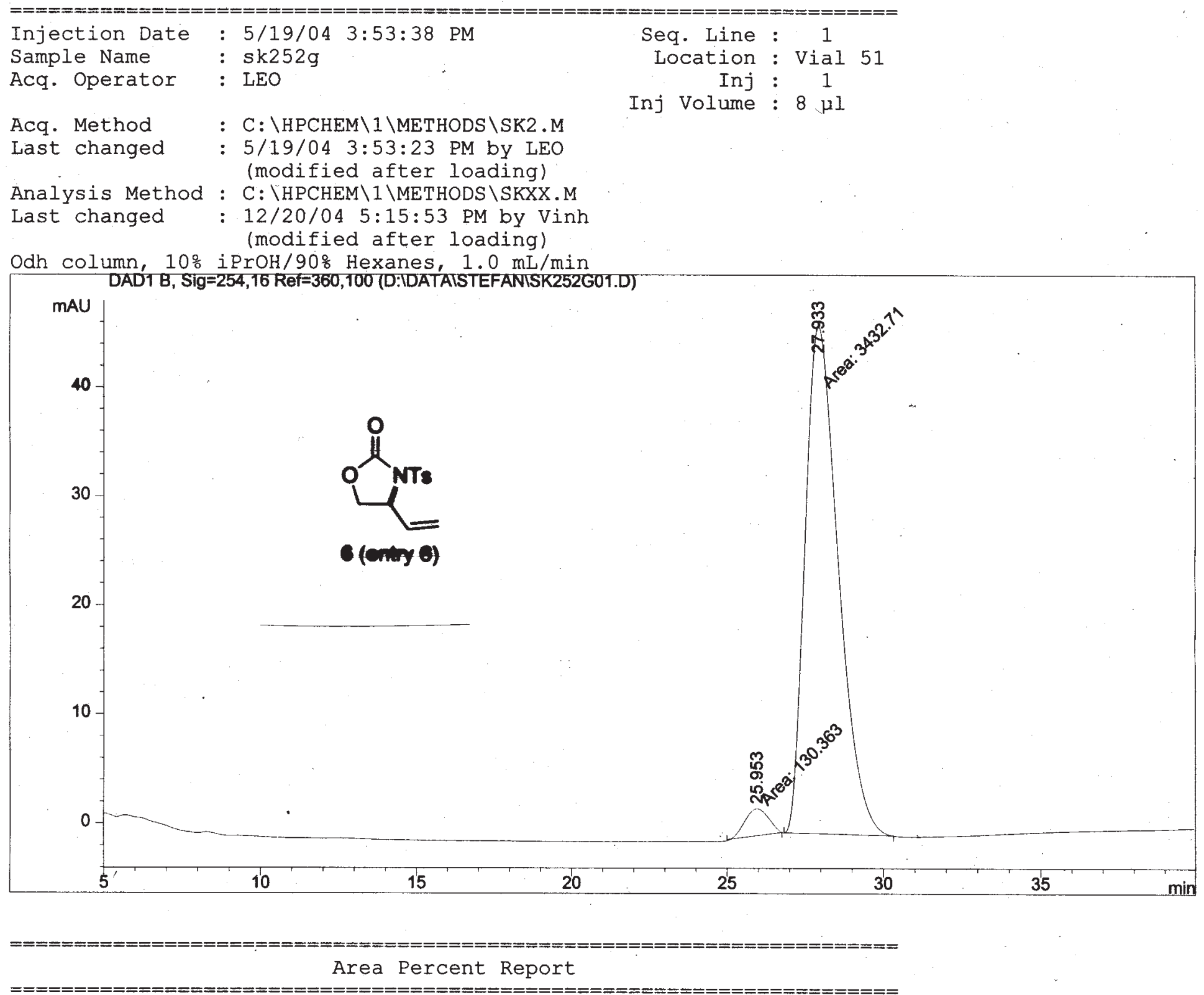

$\begin{array}{lcc}\text { Sorted By } & : & \text { Signal } \\ \text { Multiplier } & : & 1.0000 \\ \text { Dilution } & : & 1.0000 \\ \text { Use Multiplier \& Dilution Factor with } & \text { ISTDs }\end{array}$

Signal 1: DAD1 B, Sig=254,16 Ref=360,100

\begin{tabular}{|c|c|c|c|c|c|c|}
\hline $\begin{array}{c}\text { Peak } \\
\#\end{array}$ & $\begin{array}{c}\text { RetTime } \\
\text { [min] }\end{array}$ & Type & $\begin{array}{l}\text { Width } \\
\text { [min] }\end{array}$ & $\begin{array}{c}\text { Area } \\
{\left[\mathrm{mAU}^{*} \mathrm{~s}\right]}\end{array}$ & $\begin{array}{l}\text { Height } \\
\text { [mAU] }\end{array}$ & $\begin{array}{c}\text { Area } \\
\text { 응 }\end{array}$ \\
\hline -ーー- & ------ & $-\infty$ & $-\infty---$ & --------- & $=----$ & --- \\
\hline 1 & 25.953 & MM & 0.8719 & 1.30 .36264 & 2.49200 & 3.6587 \\
\hline 2 & 27.933 & MM & 1.2300 & 3432.71387 & 46.51216 & 96.3413 \\
\hline Tota & : & & & 3563.07651 & 49.00416 & \\
\hline
\end{tabular}

Results obtained with enhanced integrator!

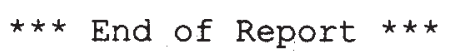




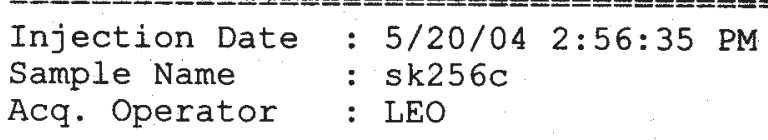

Acq. Method : C: $\backslash$ HPCHEM $\backslash 1 \backslash$ METHODS $\backslash S K 2 . M$ Last changed : 5/20/04 12:49:38 PM by LEO Analysis Method: C: \HPCHEM $\backslash 1 \backslash$ METHODS $\backslash S K X X . M$ Last changed : 12/20/04 5:17:36 PM by Vinh (modified after loading)

odh column, 108 iProH $/ 90 \%$ Hexanes, $1.0 \mathrm{~mL} / \mathrm{min}$

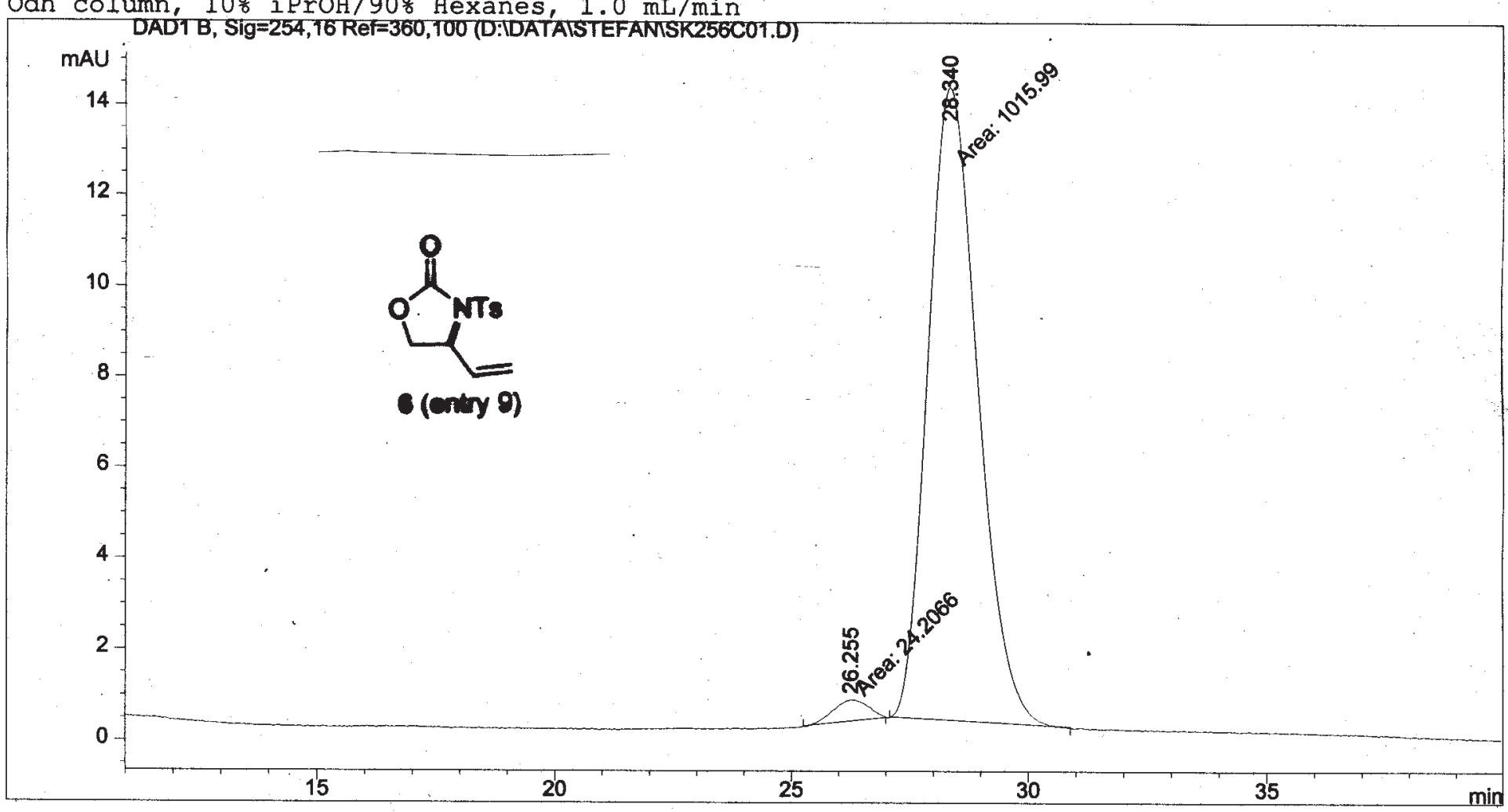

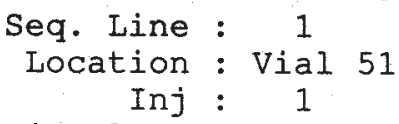

\section{Area Percent Report}

$\begin{array}{lll}\text { Sorted By } & : & \text { Signal } \\ \text { Multiplier } & : & 1.0000 \\ \text { Dilution } & : & 1.0000\end{array}$

Use Multiplier \& Dilution Factor with ISTDs

Signal 1: DAD1 B, Sig=254,16 Ref $=360,100$

\begin{tabular}{|c|c|c|c|c|c|c|}
\hline $\begin{array}{c}\text { Peak } \\
\quad \#\end{array}$ & $\begin{array}{l}\text { RetTime } \\
\text { [min] }\end{array}$ & Type & $\begin{array}{l}\text { Width } \\
\text { [min] }\end{array}$ & $\begin{array}{c}\text { Area } \\
{\left[\mathrm{mAU}^{*} \mathrm{~s}\right]}\end{array}$ & $\begin{array}{l}\text { Height } \\
\text { [mAU] }\end{array}$ & $\begin{array}{c}\text { Area } \\
\frac{0}{5}\end{array}$ \\
\hline 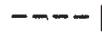 & 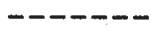 & & ------- & | - - - - - - - & ---------- & \\
\hline $\begin{array}{l}1 \\
2\end{array}$ & $\begin{array}{l}26.255 \\
28.340\end{array}$ & $\begin{array}{l}\text { MM } \\
\text { MM }\end{array}$ & $\begin{array}{l}0.8574 \\
1.2138\end{array}$ & $\begin{array}{r}24.20664 \\
1015.99005\end{array}$ & $\begin{array}{r}4.70570 \mathrm{e}^{-1} \\
13.95080\end{array}$ & $\begin{array}{r}2.3271 \\
97.6729\end{array}$ \\
\hline Tota] & : & & & 1040.19670 & 14.42137 & \\
\hline
\end{tabular}

Results obtained with enhanced integrator! 
28 IPA in Hex, AS, $1 \mathrm{ml} / \mathrm{min}$
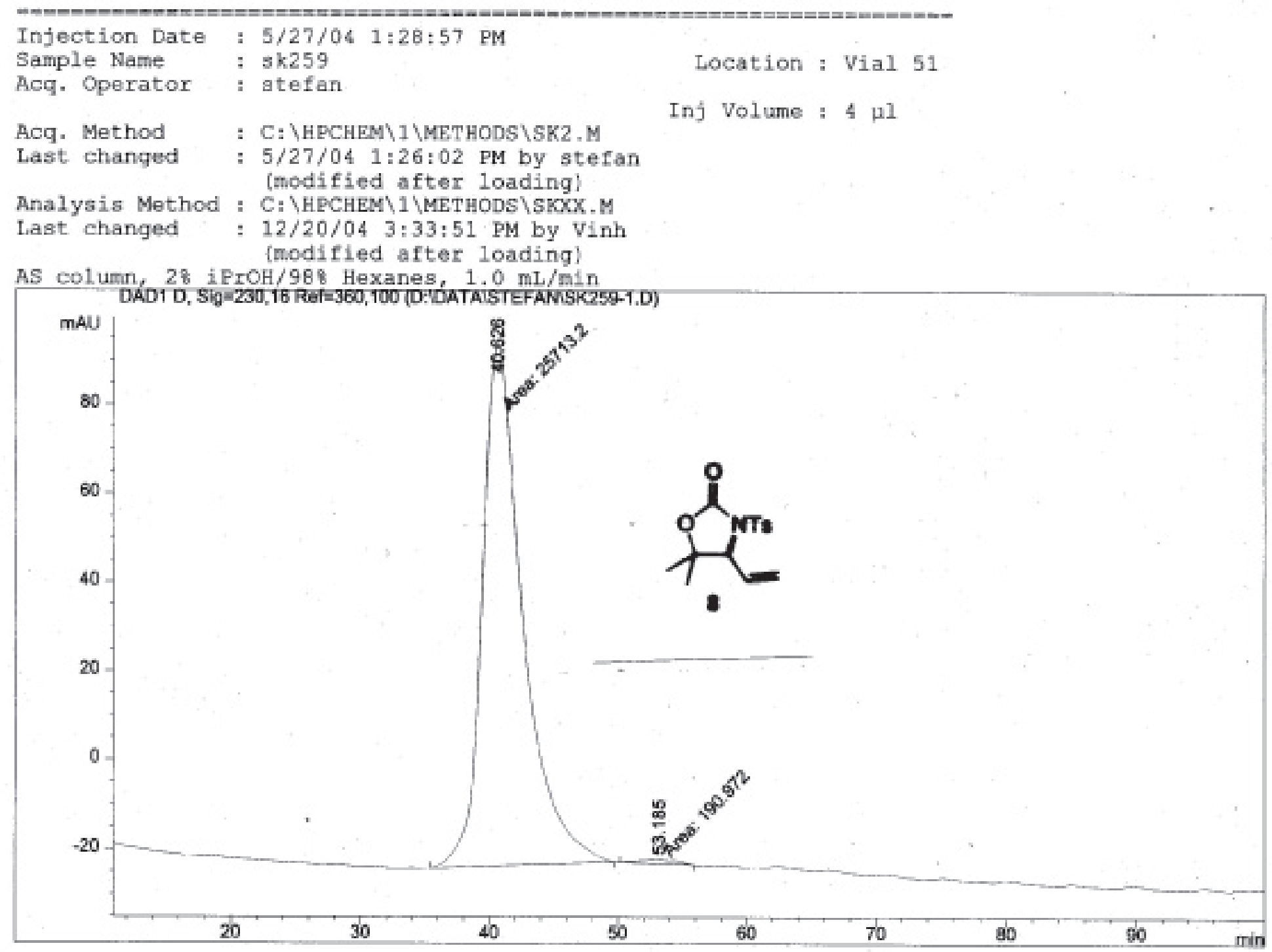

Area Percent Report

Sorted By

Multiplier

Dilution

Signal

1.0000

1.0000

Jse Multiplier \& Dilution Factor with ISTDs

Signal 1: DAD1 D, Sig=230,16 Ref $=360,100$

\begin{tabular}{|c|c|c|c|c|c|c|}
\hline $\begin{array}{c}\text { Peak } \\
\#\end{array}$ & $\begin{array}{c}\text { RetTime } \\
\text { [min] }\end{array}$ & Type & $\begin{array}{l}\text { Width } \\
\text { [min] }\end{array}$ & $\begin{array}{c}\text { Area } \\
{\left[\mathrm{m} A U^{*} \mathrm{~s}\right]}\end{array}$ & $\begin{array}{l}\text { Height } \\
\text { [mAO] }\end{array}$ & $\begin{array}{c}\text { Area } \\
8\end{array}$ \\
\hline 1 & & Mt & & 2.5 & & \\
\hline 2 & 185 & MM & & 90.97217 & $80749 e-1$ & 0.7372 \\
\hline
\end{tabular}

Totals :

$$
2.5904204 \quad 117.99279
$$

Results obtained with enhanced integrator! 
10\% IPA in Hex, OdH, $1 \mathrm{mI} / \mathrm{min}$

Injection Date : $5 / 26 / 04 \quad 4: 01: 22 \mathrm{PM}$
Sample Name

Acq. Operator: stefan

Acq. Method :C: $\backslash$ HPCHEM $\backslash 1 \backslash M E T H O D S \backslash S K 2 . M$

Last changed : 5/26/04 4:01:21 PM by stefan (modified after loading)

Analysis Method : C: \HPCHEM \1 \METHODS $\backslash S K 2 . M$

Last changed : 5/26/04 6:38:59 PM by stefan (modified after loading)

$10 \%$ IPA in Hexanes, Odh column, 1.0 ML/MIN
Location : Vial 51

Inj Volume : $8 \mu 1$

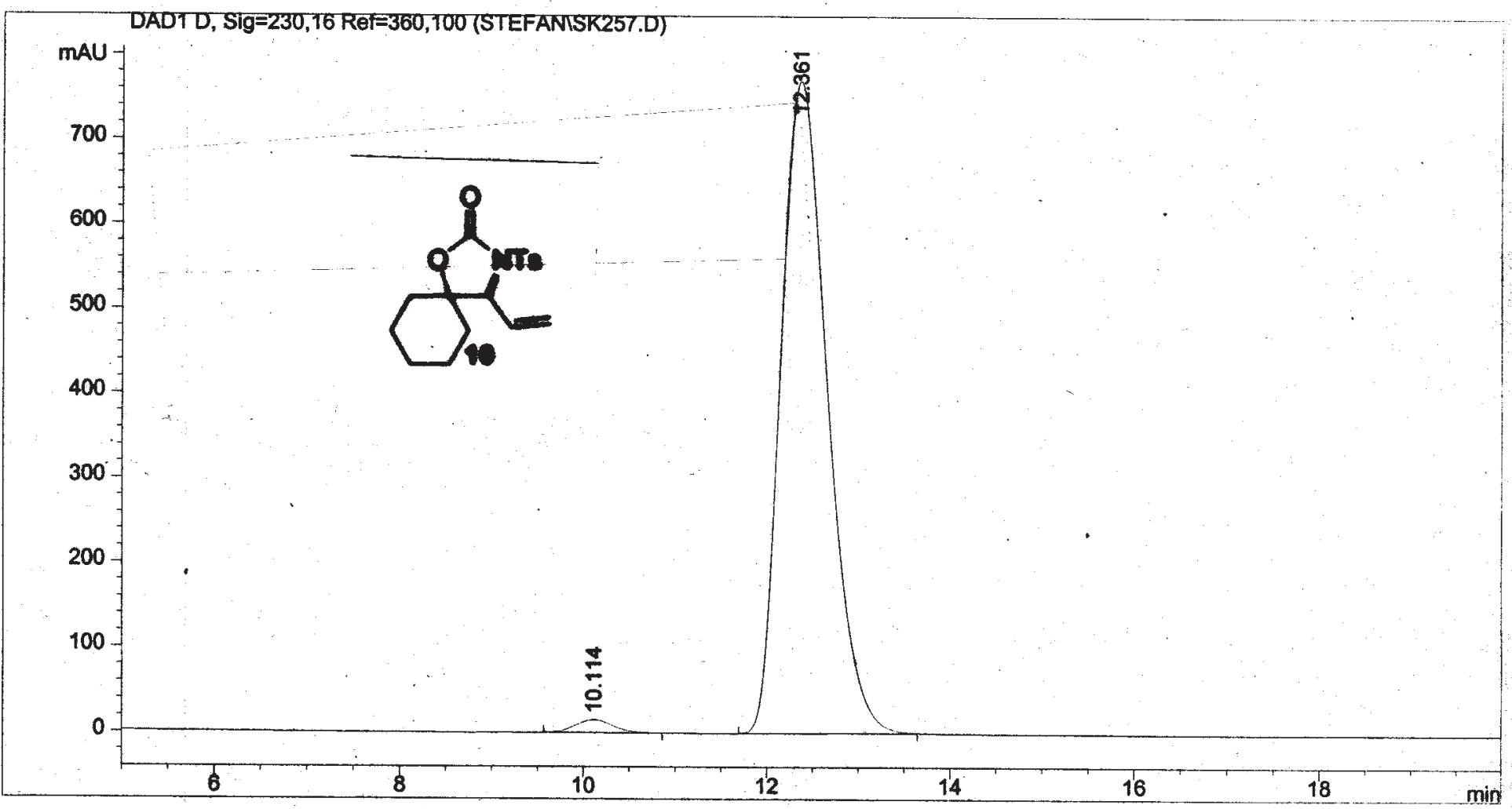

Area Percent Report

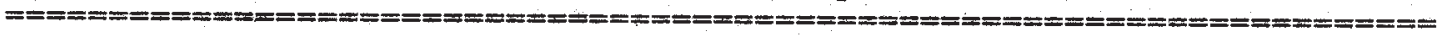

Sorted By : : Signal

Multiplier : : $\quad 1.0000$

Dilution : $\quad 1.0000$

Use Multiplier \& Dilution Factor with ISTDs

Signal 1: DAD1 D, Sig=230,16 Ref=360,100

\begin{tabular}{|c|c|c|c|c|c|c|}
\hline $\begin{array}{c}\text { Peak } \\
\#\end{array}$ & $\begin{array}{c}\text { RetTime } \\
\text { [min] }\end{array}$ & Type & $\begin{array}{l}\text { Width } \\
\text { [min] }\end{array}$ & $\begin{array}{c}\text { Area } \\
{\left[m A U^{\star} s\right]}\end{array}$ & $\begin{array}{l}\text { Height } \\
\text { [mAU] }\end{array}$ & $\begin{array}{c}\text { Area } \\
\frac{8}{8}\end{array}$ \\
\hline-4 & & & & $-\infty$ & & \\
\hline $\begin{array}{l}1 \\
2\end{array}$ & $\begin{array}{l}10.114 \\
12.361\end{array}$ & $\begin{array}{l}\mathrm{BB} \\
\mathrm{BB}\end{array}$ & $\begin{array}{l}0.4423 \\
0.5498\end{array}$ & $\begin{array}{r}442.58783 \\
2.70538 \mathrm{e} 4\end{array}$ & $\begin{array}{r}15.53955 \\
770.24597\end{array}$ & $\begin{array}{r}1.6096 \\
98.3904\end{array}$ \\
\hline
\end{tabular}

Totals : $\quad 2.74964 \mathrm{e} 4 \quad 785.78553$

Results obtained with enhanced integrator!

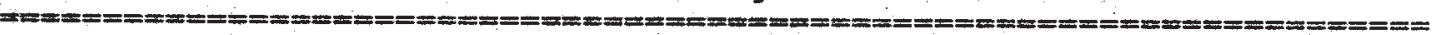




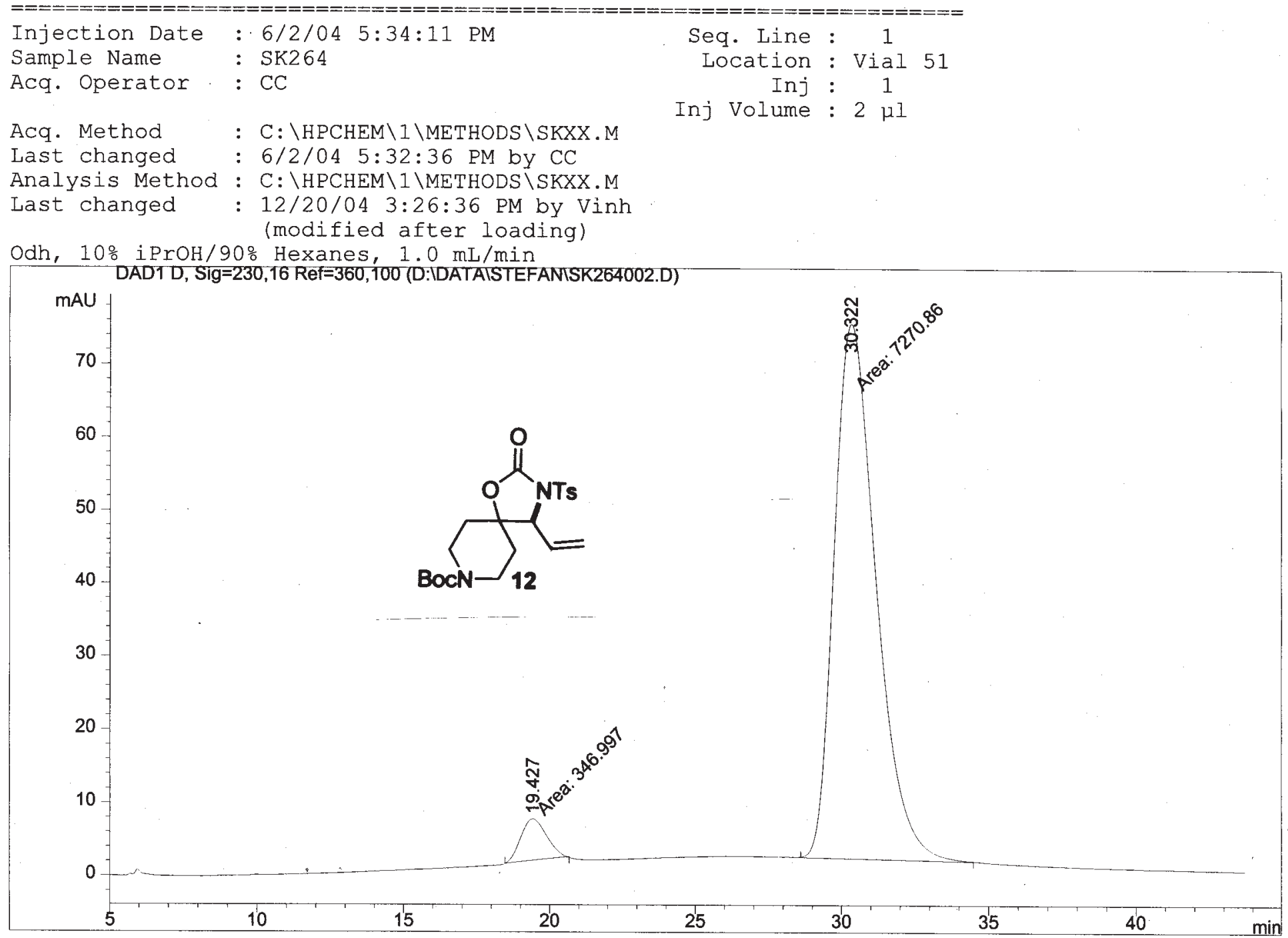

Area Percent Report

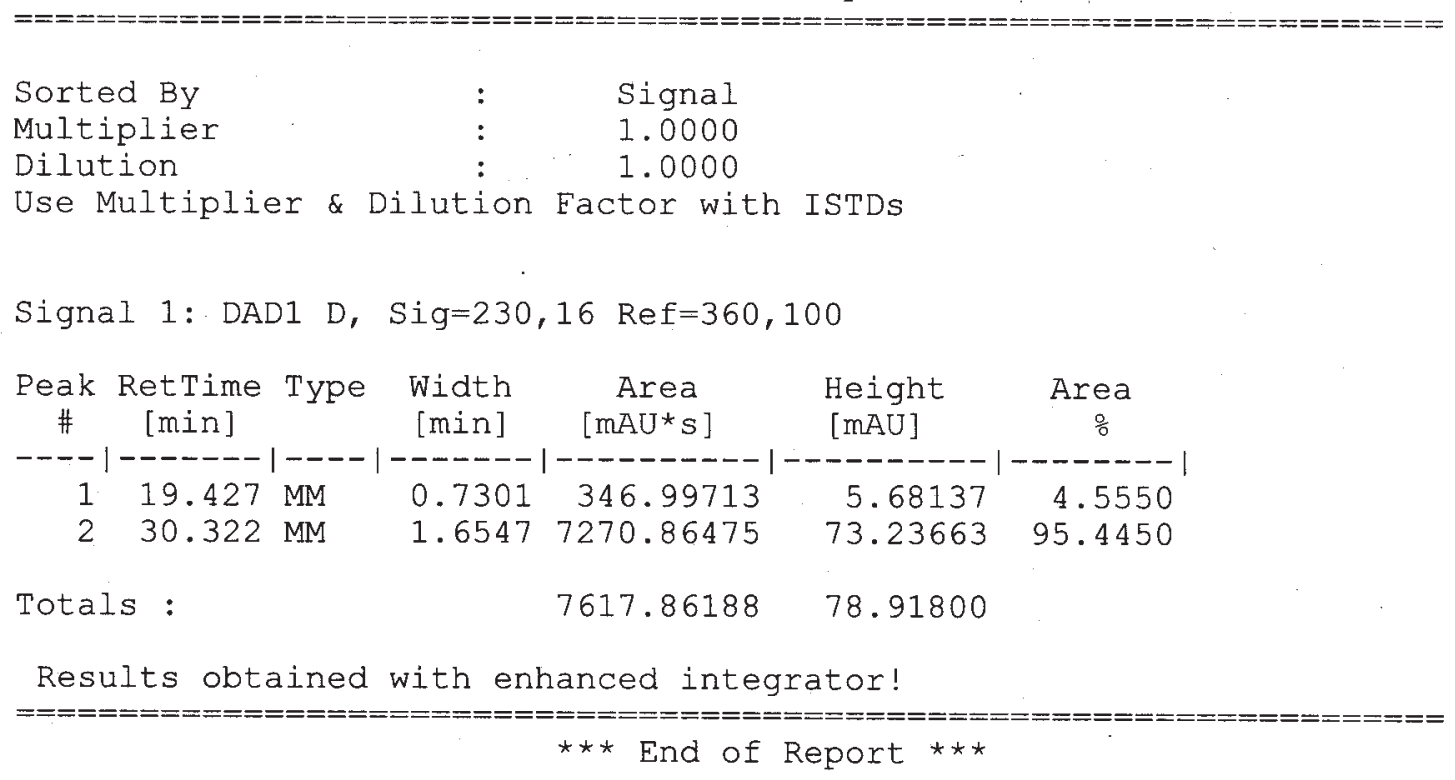

